Meklenborg, Lohndal \& Østby (eds.) Syntax, semantics and acquisition : In honor of Hans Petter OSLa Helland, Oslo Studies in Language 12(1), 2021. 145-162. (ISSN 1890-9639 / ISBN 978-82-9139812-9)

http://www.journals.uio.no/osla

\title{
NORME ET VARIATION DANS L'ENSEIGNEMENT DU FRANÇAIS LANGUE ÉTRANGÈRE : QUESTIONS DE PHONÉTIQUE
}

\author{
CHANTAL LYCHE ET KATHRINE ASLA ØSTBY \\ Université d'Oslo
}

RÉSUMÉ

La langue orale se caractérise par une multitude de pratiques. Elle ne dispose pas, comme le fait la langue écrite, d'une norme stable et objective ; la variété légitime repose sur des normes subjectives reflétant les usages de groupes socialement dominants. Dans le présent article nous nous interrogeons sur la place de la variation dans l'enseignement de la prononciation dans un contexte de français langue étrangère.

\section{[1] INTRODUCTION}

Tout enseignement d'une langue, qu'elle soit maternelle ou étrangère, présuppose une réflexion approfondie sur la notion de norme. Pour ce qui est de la syntaxe, le modèle de référence se construit sans peine sur la base des ouvrages des grands auteurs de la littérature française qui fournissent un vaste corpus aisément consultable. Dans ce vaste corpus convergent le plus souvent les trois types de norme qui interviennent dans l'enseignement d'une langue étrangère : la norme objective (une pratique régulière attestée dans un corpus donné), la norme prescriptive (le modèle à imiter) et la norme subjective (l'usage envisagé comme légitime par un locuteur). Comme le souligne Laks (2002), l'usage syntaxique invoque toujours, au moins implicitement, la notion de norme. Toute variante est analysée à l'aune de cette norme, le plus souvent caractérisée de déviance ou même de faute. Les grammaires scolaires illustrent parfaitement ce principe en proposant une norme prescriptive qui s'appuie sur maints exemples tirés de la littérature. Les ouvrages dédiés à l'enseignement du français langue étrangère (FLE) suivent la même voie, parfois de façon fort explicite, comme la grammaire de Delatour et al. (1991) où chaque chapitre clôt sur un tableau 'Ne dites pas / Dites' (par exemple p. 176 'Ne dites pas Chez la famille Grandet; Dites Chez les Grandet, Dans la famille Grandet'). Remarquons enfin que deux énoncés sont rarement équivalents, départagés par le sens, le contexte. Gadet (2007 :154) 
cite comme exemple la situation suivante : un inconnu frappe à la porte, le locuteur demande à un proche qui c'est ?, et n'obtenant pas de réponse, il poursuit qui est-ce?, question destinée au visiteur. La distance sociale entraîne ainsi une autre structure interrogative non équivalente à la première.

La situation s'avère bien différente en ce qui concerne la prononciation. Cette dernière est soumise à des variations géographiques plus ou moins stables, à maintes variations stylistiques ou individuelles qui conduisent à une plus grande tolérance et ont amené à la recommandation de modèles qui ont évolué d'époque en époque : la Cour, le Parlement, la Comédie française, mais surtout la bourgeoisie parisienne (Laks 2002). L'enseignement de la phonétique ne bénéficie pas non plus d'une solide base empirique comparable à celle de la syntaxe. Le modèle de référence a dans l'ensemble été construit à partir d'observations personnelles et il a toujours été soumis à des jugements, des évaluations individuelles où les observateurs et prescripteurs projetaient en norme prescriptive leur propre norme subjective (Morin 2000). Il faudra attendre la fin du $20^{\text {ème }}$ siècle pour voir apparaitre des corpus oraux susceptibles d'être utilisés pour l'enseignement, en particulier le corpus du programme Phonologie du français contemporain (Durand et al. 2009, Detey et al. 2010, 2016) qui nous permet de porter un regard neuf sur les choix pédagogiques à entériner.

Il n'en reste pas moins vrai que les ouvrages de phonétique en FLE s'accordent sur le groupe de référence à représenter et imiter : le français soigné de la bourgeoisie parisienne, celui des Parisiens cultivés (par exemple Fouché 1959 qui, dans bien des cas, reste une référence incontournable). À une époque où la linguistique a su mettre en exergue le caractère dynamique des pratiques langagières, l'intérêt pour l'enseignement de la variation dans ses composantes intraet inter-locuteurs ${ }^{1}$ a pris un certain essor sans pour cela affecter en profondeur les pratiques (Detey \& Racine 2012, Galazzi 2015, Falkert 2019). Nous montrerons dans un premier temps qu'à la fin du $19^{\text {ème }}$ siècle, de nombreux linguistes portaient un regard plus souple sur la norme et plus favorable à la variation. Nous nous interrogerons ensuite sur la variété de langue parlée par la classe de référence, la bourgeoisie parisienne, et sur les attitudes linguistiques de ses locuteurs, pour terminer sur la question de l'introduction de la variation dans la salle de classe et de ses limites.

\section{[2] Rappel historique : Autour de paul passy}

Le développement de la phonétique comme science au $19^{\text {ème }}$ siècle a profondément influencé l'enseignement des langues qui remet en valeur l'oral sous toutes

[1] Il s'agit donc aussi bien de la variation diaphasique, diastratique que diatopique. Dans ce travail, nous utilisons indifféremment les termes diatopique et géographique. 
ses formes et se penche particulièrement sur la prononciation. ${ }^{2}$ Jespersen n'écrit-il pas en 1902 (cité ici dans la traduction de 1904 : 145) ?:

Our pronunciation according to the old school is extremely poor, indeed, much more frightful than most people imagine. It has among others these two disadvantages, that we do not understand the natives, and that we are not understood by them.

Ce constat implacable résonne à travers l'Europe, dès 1879 chez Storm par exemple qui s'exprime en ces termes sur la situation norvégienne (Storm 1879 : 3):

Mangen brav Mand, som troede at være en hel Franskmand, bliver ved sin Komme til Frankrig ubehagelig overrasket, ved at han ikke forstaar et Ord av hvad Folk sige, og at Franskmændene heller ikke forstaa et Ord af hans Fransk! $!^{3}$

Pour remédier à cet état de fait, ce dernier propose d'introduire dans l'enseignement des dialogues illustrant la langue courante et de prendre comme modèle de prononciation la langue d'une personne éduquée, modèle qui, pour le français, se maintiendra jusqu'à nos jours avec l'ajout de la précision géographique, la langue parlée à Paris. Sweet $(1899: 42)^{4}$ considère comme souhaitable l'adoption, comme standard de prononciation, de la langue de la capitale du pays pour s'aligner sur la langue littéraire qui n'est rien d'autre que 'the written form of the older Parisian dialect'. La capitale d'un pays s'impose par le nombre de ses locuteurs, par le fait que les étrangers la visitent régulièrement, qu'elle constitue un point de départ pour leurs voyages dans le pays. Martinet \& Walter (1973) ont d'ailleurs montré pour le français que les usages de la capitale ont un pouvoir d'extension beaucoup plus puissant que celui des autres régions.

Conscient de la variation diaphasique, de la variation due à la vitesse d'élocution, Sweet propose comme objectif 'a medium colloquial style of pronunciation' (Sweet 1899: 41). Cette vitesse moyenne se caractérise par exemple selon lui pour le français par la prononciation '(estrordinęr) (si vu $\mathrm{pl \varepsilon})^{{ }^{5}}$ pour

[2] Pour un aperçu de l'évolution de cet enseignement, voir par exemple Puren (1988), Germain (1993), Besse (2012).

[3] De nombreuses braves gens qui croyaient être des Français authentiques, sont à leur arrivée en France désagréablement surprises du fait qu'ils ne comprennent pas un mot de ce que disent les gens, et de ce que les Français ne comprennent pas non plus un mot de leur français (notre traduction CL/KAØ).

[4] Dans la préface, Sweet précise que la première version de ce travail date de 1877. Henry Sweet était très proche de Johan Storm, à qui il rendait régulièrement visite. C'est en Norvège qu'il aurait achevé son ouvrage et il reconnaitra que Storm "had provided "the main impulse" for his interest in the reform of language teaching methodology' (Howatt \& Widdowson 2004).

[5] Sweet $(1899: 41)$ reprend ces exemples à Passy. 


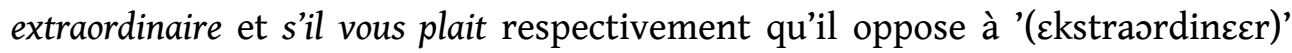
dans un style plus lent et '(sj u ple)' pour un style rapide. Ces remarques montrent à quel point les descriptions de l'oral il y a plus d'un siècle savaient se détacher de l'écrit et mettaient à l'honneur un registre de langue courante. On notera dans la foulée que l'ouvrage de Bødtker \& Høst (1896) ${ }^{6}$ destiné à l'enseignement du français dans les écoles norvégiennes adoptait ce style de conversation. On y mentionne par exemple la chute de la liquide dans le groupe final obstruante + liquide (à table [a tab]), la chute de la liquide du pronom personnel devant consonne (il part [i par]), [i ja] pour il y a (Henriksen \& Lyche 1997). ${ }^{7}$ Il est donc possible d'affirmer qu'un enseignement de l'oral que l'on pourrait qualifier de moderne, explose littéralement en Europe et aux États-Unis à la fin du $19^{\text {ème }}$ siècle, soutenu par un grand mouvement réformateur qui a donné naissance à plusieurs méthodes dont la méthode dite imitative, ${ }^{8}$ très présente en Norvège sous l'influence de Storm, et ce que l'on a appelé la méthode directe (Howatt \& Smith 2002, Besse 2012, Durand \& Lyche sous presse) dans de nombreux pays européens.

Paul Passy (1851-1940), avocat inconditionnel d'une réforme de l'orthographe, grand défenseur de l'oral, cheville ouvrière dans le développement de l'alphabet phonétique international et dans celui de la méthode directe, mérite une mention particulière dans ce paysage (Durand \& Lyche 2019, sous presse). Son ouvrage, Les sons du français, qui connaîtra 12 éditions (de 1887 à 1932), augmentées et remaniées jusqu'à la septième, nous intéressera spécialement puisqu'il fournit des spécimens transcrits en alphabet phonétique. Dans la deuxième édition, de 1889, il précise le style d'élocution que ces spécimens sont sensés illustrer. Il reconnaît (p. 78) que le lecteur pourrait éprouver quelque difficulté 'a déchifrer ce grimoire', ${ }^{9}$ et que la prononciation décrite ne correspond pas à la prononciation 'téoriquement corecte' recommandée par les grammaires. Passy est conscient que la prononciation indiquée pourrait être jugée vulgaire, mais il recommande au lecteur de lire plusieurs fois les textes à haute voix pour se rendre compte de ce qu'en fait, il s'agit tout simplement de la 'prononsiacion uzuèle, employée par les jans cultivés lorsqu'ils n'y font pas atansion' (p. 78). Dans l'introduction aux spécimens, Passy avertit le locuteur que du fait de la variation constante de la prononciation d'un locuteur à un autre et du fait des difficultés rencontrées par chacun à rendre compte objectivement de sa propre

[6] Voir Henriksen (2004) pour une analyse de toutes les éditions.

[7] Il faut ici voir l'influence de Storm, lui-même fortement influencé par Paul Passy qu'il questionne régulièrement sur des faits de prononciation (Durand et Lyche 2019, sous presse).

[8] Cette méthode, à l'opposé de la méthode directe, maintient la traduction en début d'apprentissage pour aider l'apprenant, elle propose la transcription phonétique en parallèle à la transcription orthographique. Elle s'appuie sur la répétition en chœur (Henriksen et Lyche 1997).

[9] Nous reproduisons fidèlement ici l'orthographe utilisée par Passy dans ses écrits. 
prononciation, les transcriptions qu'il propose peuvent paraître étranges à de nombreux lecteurs. Il démontre fort bien l'écart susceptible d'exister entre la norme subjective et la norme objective chez des personnes cultivées comme l'était son père, ${ }^{10}$ à l'aide de l'anecdote suivante rapportée dans Les sons (1906 : 139) :

Quand O. Jespersen était en France, mon frère Jean et moi lui citions des formes caractéristiques du français parlé. Mon père qui nous écoutait, protestait énergiquement; il ne voulait pas admettre, notamment, que il se prononce (i) devant les consonnes. Comme nous insistions, il a fini par s'écrier : (mœsjø jespersen, i n sav pa s k i di:z) ; montrant ainsi, bien malgré lui, que nous avions raison. [Je lui ai souvent entendu dire (i), même dans une conférence publique, quand il s'animait.] Plus récemment, il me reprochait d'avoir noté, dans mes textes, parce que par (paskə) : 'ce qui est très fautif, disait il (paskə õn di pa paskə).'

En 1906, Passy regroupe les textes en transcription phonétique sous trois étiquettes reflétant trois styles de langue : la prononciation familière ralentie, la prononciation soignée et la prononciation très soignée. Il ajoute en 1913 un quatrième registre, la prononciation familière rapide et c'est également dans l'introduction de cette édition qu'il se prononce pour la première fois sur la notion de norme et propose comme modèle (Passy 1913 : 9) :

[L]a prononciation des personnes cultivées du Nord de la France plus spécialement, celle de la région parisienne - plus spécialement encore, la prononciation naturelle de l'auteur, normalisée dans un petit nombre de cas. Rien, à vrai dire, ne nous autorise à dire que cette prononciation soit la meilleure. Mais nous pouvons affirmer sans crainte qu'elle sera partout reconnue comme acceptable, comme correcte. ${ }^{11}$

Comme norme pour l'enseignement, il recommande, comme Sweet avant lui, 'la prononciation familière ralentie', ${ }^{12}$ même si, écrit-il, 'À un point de vue abstrait, chaque variété peut être regardée comme également bonne ; mais pour l'enseignement, on est obligé de faire un choix' (Passy 1913 : 9). Les deux exemples suivants (Passy $1892: 128$ ), illustrent ce niveau de langue et témoignent d'un niveau

[10] Frédérique Passy fut le premier lauréat du prix Nobel de la Paix avec Henry Dunant.

[11] Ces propos sont repris dans l'introduction à son French phonetic reader de 1914 : 'this "educated Northern pronunciation" cannot claim any intrinsic superiority over others'.

[12] En italiques dans le texte. 
élevé d'élisions : 'i rgard a se pje e i vwa' (il regarde à ses pieds et il voit) ' $\mathrm{j}$ a rj $\tilde{\varepsilon}$ ki rõ osi œrø k d \&t bõ' (il n'y a rien qui rend aussi heureux que d'être bon).

Passy se démarque profondément de ses contemporains et de ce que l'on pourra lire dans la plupart des manuels écrits au $20^{\text {ème }}$ siècle par ses remarques sur la variation stylistique et géographique. Son intérêt pour les dialectes le conduit à proposer des transcriptions dans plusieurs variétés de français (Nord, Midi, Suisse) auxquelles il ajoute à partir de 1917 la parabole de l'enfant prodigue dans trois patois (Normandie, Vosges, Hautes-Pyrénées). Comparé à Grammont (1914) ou Fouché (1959), Passy s'affirme ainsi comme un auteur très moderne. Rappelons que Grammont ne tolérait aucune entorse à une vue très restrictive de la norme (1914:1) :

Cet ouvrage est destiné essentiellement aux étrangers et aux provinciaux qui veulent se perfectionner dans la bonne prononciation française ou se renseigner sur elle. Toutes les personnes compétentes reconnaissent aujourd'hui que cette prononciation française est celle de la bonne société parisienne, constituée essentiellement par les représentations des vieilles familles de la bourgeoisie. C'est celle-là qu'on s'est efforcé de décrire dans ce traité.

Si Passy semble bien le seul avocat en France d'une certaine pluralité linguistique dans l'enseignement, sa position nuancée sur la norme se retrouve hors de France, particulièrement dans les pays scandinaves, chez les membres de l'organisation Quousque tandem qu'il rencontre au Congrès de Stockholm en 1886 (Durand \& Lyche 2019). Le manuel de français que Kristoffer Nyrop publie en 1893 et où il reconnaît sa dette envers Passy, ${ }^{13}$ présente à l'apprenant diverses prononciations régionales, avec un intérêt particulier pour le français du midi, et s'abstient de définir une norme prescriptive. Selon Nyrop, les transcriptions proposées dans son ouvrage ne sont pas les seules légitimes et par ailleurs, l'usage d'une même personne n'est jamais fixe, il est sujet à de constantes variations. Nyrop réitère avec force cette opinion dans la traduction française de son manuel et ajoute même (1902: VII) :

Mais le bon usage, on l'a déjà dit, ressemble parfois singulièrement à la fée Morgane qui s'évanouit en fumée quand on s'en approche de trop près. ${ }^{14}$

[13] Il remercie Johan Storm, Paul Passy et Otto Jespersen dans cet ordre.

[14] Nyrop reprend ici les propos d'Eduard Koschwitz : 'sans jamais pouvoir saisir cette fée Morgane qui, nécessairement, se dissout en nuées dès qu'on s'en approche de trop près.' (1896: II). Il est intéressant de noter que la mise en question de l'existence réelle d'un bon usage apparaît exclusivement chez des étrangers (Koschwitz est allemand). 
Pourquoi aussi vouloir établir un seul bon usage quand la pratique nous apprend tous les jours qu'il y en a plusieurs? En matière d'orthoépie il est bon de combattre la manie si répandue d'établir des dogmes; aussi avons-nous souvent admis comme également bonnes deux, même trois ou quatre manières de prononcer un mot.

Ces ouvertures vers une conception plurielle de la norme n'ont guère été suivies et la plupart des manuels de FLE maintiennent une idéologie du standard très proche des recommandations de Grammont. Mais quelle est la réalité langagière de ce groupe que Grammont et bien d'autres prennent comme modèle, que saiton vraiment des pratiques des 'vieilles familles de la bourgeoisie' ? S'agit-il vraiment d'un groupe d'une telle homogénéité que l'on peut extraire de leurs discours des règles de bonne prononciation de la même façon que l'on propose des règles pour la morphologie et la syntaxe ? Et comment une telle 'norme' est-elle appréhendée par les locuteurs concernés?

[3] LA BOURGEOISIE PARISIENNE : CLASSE DE RÉfÉRENCE ET ATtTitudes LINGUISTIQUES

La bourgeoisie parisienne, qui a constitué pendant tout le $20^{\text {ème }}$ siècle le groupe de référence en matière de prononciation, n'a que très rarement été l'objet d'étude des travaux (socio)linguistiques empiriques. On trouve néanmoins dans Østby (2016) une étude approfondie aussi bien des pratiques que des attitudes linguistiques de 6 femmes et 6 hommes âgés de 23 à 86 ans appartenant (par autoidentification) à ce groupe. ${ }^{15}$

Dans les études d'inspiration variationniste, la notion de classe sociale joue un rôle central pour rendre compte de la variation linguistique. Les travaux classiques de Labov $(1966,1972)$ privilégient l'étude des classes dominées, dont les usages se caractérisent dans l'ensemble par l'emploi de peu de formes de prestige et de nombreuses formes non standard, contrairement aux pratiques des classes socialement dominantes. Les locuteurs appartenant aux classes moyennes et inférieures exhibent en outre un niveau d'insécurité linguistique élevé. La notion d'insécurité linguistique, introduite dans Labov (1966), fait référence à un manque de légitimité linguistique vécu par le locuteur. Labov propose de mesurer ce phénomène en comparant ce que le locuteur rapporte correspondre à son usage personnel (cf. norme objective) et ce qu'il estime correspondre à l'usage correct (cf.

[15] Le travail de Østby (2016) repose sur un travail de terrain réalisé à Paris entre 2004 et 2006 dans le cadre du projet Phonologie du français contemporain. Pour documenter les pratiques linguistiques des locuteurs, le protocole d'enquête du projet (Durand \& Lyche 2003), d'inspiration labovienne, a été adopté, et ce protocole a été complété par un second questionnaire afin d'examiner les attitudes, notamment le niveau de sécurité et de purisme linguistiques, du groupe. 
norme subjective). Un écart entre les deux est interprété comme un signe d'insécurité linguistique, allant typiquement de pair avec une perception erronée de ses propres productions, une dépréciation des usages du groupe auquel on appartient ainsi qu'un effort conscient de correction pour s'approcher d'un modèle de prestige extérieur. La condamnation prononcée par ces locuteurs des formes estimées fautives, non seulement dans leur usage personnel mais aussi dans les usages des autres, est de surcroît considérée par Labov comme du purisme linguistique.

Étant donnés la définition de l'insécurité linguistique telle qu'elle a été développée par Labov, ainsi que le principe que les couches supérieures sont linguistiquement conservatrices, Østby (2016) propose l'hypothèse suivante concernant son petit échantillon de locuteurs parisiens : les locuteurs appartenant à un groupe socialement privilégié exposent des pratiques linguistiques proches de la norme de référence ainsi que des niveaux d'insécurité et de purisme linguistiques très bas.

Cette hypothèse est testée à l'aide des variables vocaliques /A, E, Ø, O/. Pour le français hexagonal, la littérature rapporte une simplification du système vocalique due à une perte progressive des oppositions phonémiques (Armstrong 2001, Armstrong \& Pooley 2010 inter alia). Ces tendances sont reflétées dans les données de Østby (2016), lesquelles indiquent ainsi des usages à la fois variables et parfois relativement innovateurs au sein de la bourgeoisie parisienne. Les tendances innovatrices se manifestent toutefois à divers degrés selon l'âge des locuteurs et selon la voyelle : pour ce qui est des voyelles ouvertes, les seniors, en particulier les hommes, exhibent un système conservateur où deux phonèmes ouverts /a/ et /a/ s'opposent, tandis que les juniors ne réalisent qu'une variante antérieure, adoptant ainsi un système simplifié. En revanche, bien que pour les voyelles moyennes les données fassent également état de variations générationnelles, les oppositions phonémiques entre la série des voyelles mi-fermées [e, $\varnothing$,

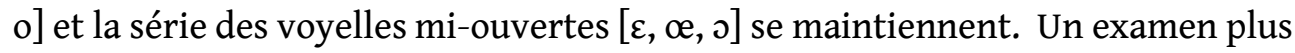
détaillé révèle en outre des variations individuelles non négligeables pour toutes les variables dans les propos des locuteurs. Les données montrent donc que si le système vocalique de ces locuteurs présente en effet les caractéristiques de la norme pédagogique classique (Fouché 1959), il est néanmoins fluctuant et reste empreint de variations.

Pour ce qui est des attitudes de ces mêmes locuteurs, les calculs de l'index d'insécurité linguistique (IIL, cf. Index of Lingustic Insecurity, ILI, Labov 1966 : 96) reflètent un niveau de sécurité linguistique très élevé. L'IIL est calculé de la manière suivante ${ }^{16}$ : pour un ensemble de mots comportant les variables examinées,

[16] Voir Østby (2016) pour une description plus détaillée des calculs de l'IIL ainsi que du taux de purisme. 
les témoins sont invités à sélectionner, parmi plusieurs variantes possibles, la forme qui correspond à leur propre prononciation ainsi que la forme correcte. $\mathrm{Si}$ les deux diffèrent, le témoin reçoit un 'point' ; les mots étant au nombre de 21, l'IIL maximum, indiquant un cas d'insécurité extrême, est également 21. Pour son échantillon parisien, Østby (2016) obtient un IIL moyen de 1,6, confirmant ainsi l'hypothèse d'une situation d'extrême sécurité linguistique. Une comparaison entre les auto-évaluations et les données de production des témoins montre d'ailleurs que ces derniers ont une perception très précise de leurs pratiques réelles, contrairement à ce qui était le cas dans l'anecdote de Passy (section 2). Le sentiment de sécurité, ou légitimité, linguistique ressort également des données épilinguistiques : à la question 'Si vous hésitiez pour une prononciation, qui choisiriez-vous comme modèle de prononciation?', les témoins répondent par exemple 'Je n'hésite pas, je sais comment il faut prononcer', mettant de surcroit en valeur leur propres usages parce que parisiens dans une réponse comme 'Pour la forme correcte, j'aurais tendance à dire la mienne, car je suis parisienne'.

Confirmant également son hypothèse, Østby (2016) observe un niveau de purisme très bas au sein du groupe. Le taux de purisme a été calculé à partir du même questionnaire d'autoévaluation qui a été utilisé pour calculer l'IIL. Pour chaque mot, le témoin a été invité à sélectionner la forme la plus incorrecte selon lui et à indiquer si une telle prononciation constitue une faute légère ou grave. Pour chaque faute grave, le témoin reçoit un 'point', et le nombre de points détermine le niveau de purisme relatif du témoin (Ledegen 2000). Un taux de purisme bas signifie donc que les locuteurs ne voient pas une prononciation incorrecte comme une faute grave; les commentaires des locuteurs indiquent que seules sont considérées comme graves les erreurs qui risquent d'entraver la communication. On constate donc pour cet échantillon parisien dans son ensemble une attitude relativement laxiste, ouverte à la variation.

Le cas des voyelles de type A est particulièrement intéressant à cet égard : cette variable affiche l'IIL le plus élevé et en même temps le taux de purisme le plus bas. En effet, lorsque les locuteurs estiment que la forme correcte est une autre forme que celle qu'ils rapportent utiliser eux-mêmes, ils considèrent en général leur prononciation déviante comme une erreur légère. Autrement dit, la variation semble être d'autant plus acceptée qu'elle est présente. Cette étude au sein de la bourgeoisie parisienne met ainsi à jour un autre patron que celui qui se dessine traditionnellement dans les travaux variationnistes visant des locuteurs socialement dominés, où un IIL élevé est accompagné d'un taux de purisme également élevé, ce que l'on peut interpréter comme le reflet de la reconnaissance et de la mise en valeur d'une norme extérieure. Les témoins de Østby semblent se caractériser dans l'ensemble par une situation de sécurité linguistique 
tellement forte que lorsque leur propre usage dévie de leur norme subjective, ils acceptent toutes les variations, le taux de purisme tendant vers zéro.

L'étude de Østby (2016) confirme donc les observations de Nyrop, pour qui 'un seul bon usage' est aussi insaisissable que la fée Morgane. Au sein de la bourgeoisie parisienne, le groupe de référence de Grammont et bien d'autres linguistes, les pratiques linguistiques varient. Il convient également de souligner la perception positive de la variation dans ce groupe de la haute bourgeoisie parisienne, traditionnellement considéré comme conservateur, dans la mesure où une telle attitude laxiste permet l'adoption de nouvelles variantes. Ceci nous amène à reposer la question du groupe de référence, et notamment de la réalité d'une 'norme' de prononciation stable et uniforme, ainsi que celle de la place de la variation dans l'enseignement de la prononciation du français langue étrangère aujourd'hui.

\section{[4] Peut-on enseigner la VARiation dans un Contexte de fle ?}

Telle est la question posée par Paternostro (2017) qui souligne la problématique que nous avons développée ci-dessus : '[C]omment, en effet, concilier la description de la langue et de ses variations avec une vision normative prônant l'enseignement de « la » langue française, pure, homogène, immuable ?' (Paternostro 2017 : 281). Pour ce qui est plus précisément de la variation diatopique, Detey \& Lyche (2019) se penchent sur cette vision normative qui perdure chez les enseignants et les étudiants dans deux pays où cohabitent dans la sphère linguistique plusieurs variétés de langues : la Norvège et le Japon. On sait par exemple que la coexistence et la valorisation des dialectes font de la Norvège un large creuset linguistique au sein duquel la population est constamment exposée à une variété autre que la sienne. Les Norvégiens maintiennent un niveau d'inter-compréhension fort élevé et il est reconnu (Gooskens \& Heeringa 2014) qu'ils ont plus de facilités que leurs voisins danois et suédois à comprendre les autres langues scandinaves (danois, suédois et norvégien), très proches typologiquement. En ce qui concerne l'enseignement du norvégien langue étrangère, les textes officiels restent vagues sur la variété à enseigner. La prononciation des apprenants doit être norvégienne sans guère plus de précisions (Husby 2015). L'accent est cependant mis sur l'inter-compréhension. Dans l'ensemble, afin de faciliter la tâche des apprenants, du fait de sa plus grande visibilité et de la pénurie d'ouvrages qui décrivent les divers dialectes, le standard østnorsk est enseigné à l'oral, le plus souvent coloré par la prononciation dialectale de l'enseignant. Très vite, cependant, les apprenants sont soumis à des exercices de perception afin de les entraîner à comprendre d'autres variétés dialectales que la variété locale. Le multilinguisme du pays, la position de choix tenue par tous les dialectes et les 
avantages reconnus que cela confère à la population laisseraient préjuger d'un grand intérêt porté aux différentes variétés de langue dans les langues étrangères enseignées sur le territoire et c'est en effet le cas de l'anglais mais pas du français.

L'anglais bénéficie d'une situation privilégiée en étant la seule langue étrangère obligatoire pour toute la scolarité avec un enseignement qui débute dès l'entrée en primaire. Les directives officielles de 2020 (Kunnskapsdepartementet 2020) englobent dans les aptitudes langagières les capacités à communiquer dans des situations formelles et informelles, à pouvoir aborder un vaste éventail de sujets avec des interlocuteurs d'origines géographiques et sociales diverses. L'accent est mis sur le développement de la compréhension orale pour le traitement de la variation géographique qui est introduite de façon systématique au collège. Rien de tel en ce qui concerne le français pour lequel seule la dimension culturelle de la francophonie est envisagée. Amundsen (2015) souligne par ailleurs la réticence des enseignants à exposer leurs élèves à autre chose que le bon français de la région parisienne et leur totale absence de formation pour une approche plurielle, excluant ainsi toute variation intra- ou inter-locuteurs.

Comme le rappelle Valdman (2000: 648), 'L'objectif déclaré de l'approche communicative est l'acquisition de la part des apprenants d'une maîtrise quasinative de la langue cible'. Seule une sensibilisation précoce à la variation sous toutes ses formes permet d'atteindre cet objectif. Et même s'il est aujourd'hui courant de remettre en question le statut-cible du locuteur natif, en particulier en production, il n'en reste pas moins que la variation est cosubstantielle à la parole : dès que l'input n'est plus pédagogiquement construit, l'apprenant y est confronté, y compris à travers les nouveaux médias. Ainsi, l'appropriation de la compétence sociolinguistique, qui inclut une capacité de traitement de la variation diatopique, ne saurait être reléguée à la fin de l'apprentissage. Aussi, en accord avec Auger \& Valdman (1999 : 408) ('If we truly wish to acquaint American learners of FFL with the linguistic particularisms of non-Hexagonal francophones [...], we must begin early on, even in beginning secondary school courses.'), nous argumentons qu'une exposition plus longue s'impose à nos yeux. Dans une optique où l'acquisition d'une langue étrangère se conforme en partie à celle de la L1, et qu'une prononciation quasi-native peut être atteinte par des apprenants plus âgés (Birdsong 2007), la question de l'input s'avère cruciale : 'Thus the linguistic input emerges as a key factor (maybe even the key factor) in the answer to the fundamental question of L1-L2 differences.' (Slabakova 2013 : 57). Le plus souvent, les apprenants de L2/L3 n'ont accès dans le contexte scolaire qu'à un input peu varié, la voix de leur enseignant et celle des documents sonores de la méthode étudiée, épurée de tout particularisme régional ou social, 
alors qu'un input riche peut favoriser la catégorisation segmentale, la variation phonétique présente dans la distribution de cet input facilitant l'acquisition des catégories phonémiques (Lively, Logan \& Pisoni 1993, Thomson 2012).$^{17}$ Il ne s'agit cependant pas de se fixer comme objectif la maîtrise par l'apprenant de variétés distinctes, mais bien de le préparer à interagir avec des locuteurs natifs, auquel cas l'accent est mis sur la perception et non sur la production. De nombreuses voix s'élèvent pour une meilleure intégration de la variation dans la classe de langue favorisée entre autres par l'accès aux corpus oraux qui se prêtent par exemple à des exercices de transcodage (Paternostro 2017), ou par l'étude de textes issus de la communication électronique (Wachs 2017). L'introduction de la variation diatopique présente dans la francophonie par exemple, ne saurait se concevoir que de façon didactiquement raisonnée (Detey 2009) à l'aide de documents didactisés selon les niveaux de langue, comme par exemple les modules TUFS. ${ }^{18}$ Tous ces exercices entraînent l'apprenant à réfléchir sur les différentes modalités d'expressions, sur les particularités de l'oral, faisant de lui un véritable acteur dans le processus d'apprentissage (Halté 1992).

\section{[5] CONCLUSION}

La variation, omniprésente dans la L1 de l'apprenant ne saurait être éliminée de la L2 sous prétexte de complexité didactique et cognitive. Nous avons vu que tel n'était pas le cas à la fin du $19^{\text {ème }}$ siècle chez le grand pédagogue qu'était Passy, conscient des variantes stylistiques et bienveillant à l'égard des variétés dialectales. Nous avons également souligné que le groupe de référence adopté par bien des auteurs de manuels, soit la haute bourgeoisie parisienne, ne formait pas un groupe linguistique uniforme qui permettrait de définir un modèle précis et rigoureux et qu'il était lui-même ouvert à d'autres pratiques. Eu égard à l'extrême mobilité des jeunes et à l'input complexe offert par les outils numériques, 'l'apprenant en français langue étrangère doit être armé contre les fantômes idéologiques d'une normativité homogène et figée ; l'input à des fins d'exposition à la parole dans toutes ses variations est essentielle dès les premiers niveaux en langue cible' (Weber 2019: 9). Cette exposition, toute nécessaire soit-elle, sera, dans un premier temps, introduite à doses homéopathiques contrôlées en perception, suivant en cela les descripteurs du CECRL (Cadre européen commun pour les langues) :

A2 : Peut comprendre les points importants d'une histoire et se débrouiller pour suivre l'intrigue, à condition qu'elle soit racontée

[17] Voir également Clopper \& Pisoni (2004) pour la reconnaissance dialectale en L1.

[18] http://www.coelang.tufs.ac.jp/mt/fr/index_en.html 
lentement et clairement.

B1 : Peut comprendre l'information contenue dans la plupart des documents enregistrés ou radiodiffusées, dont le sujet est d'intérêt personnel et la langue clairement articulée.

$\mathrm{C} 1$ : Peut comprendre une gamme étendue de matériel enregistré ou radiodiffusé, y compris en langue non standard et identifier des détails fins incluant l'implicite des états d'esprit et des relations entre interlocuteurs.

(CECRL 2018, volume complémentaire, p. 62)

Pour ce qui est de la variété à enseigner en production, il s'agit uniquement d'un choix socio-éducatif qui évoluera selon les pays et les contextes. Au sein d'une pluralité de modèles, l'apprenant fera dans un tout premier temps un choix guidé qui favorisera l'acceptabilité et l'intelligibilité. À un niveau plus avancé, sa prononciation tendra en tous points vers celle du modèle cible avec tous ses niveaux de variation. Si jusqu'à présent la variation a été minimalisée ou inexistante dans les cours de FLE, il importe d'enterrer la notion de bon français, mythe qui peut être déconstruit grâce aux corpus oraux et aux données numériques qui aident le maitre à proposer un enseignement adapté au contexte du $21^{\text {ème }}$ siècle. Non seulement un travail de réflexion socio-phonétique s'impose dans les manuels, mais la formation des maîtres ne saurait se passer d'un dialogue étroit entre chercheurs et enseignants.

\section{RÉFÉRENCES}

Amundsen, Elin S. 2015. La variation diatopique dans l'enseignement du français en Norvège. Mémoire de maîtrise. Université d'Oslo.

Armstrong, Nigel. 2001. Social and Stylistic Variation in Spoken French. A Comparative Approach. Amsterdam : John Benjamins.

Armstrong, Nigel \& Pooley, Tim. 2010. Social and Linguistic Change in European French. Basingstoke : Palgrave Macmillan.

Auger, Julie. \& Valdman, Albert. 1999. Letting French students hear the diverse voices of Francophony. The Modern Language Journal 83 : 3, 403-412.

Besse, Henri. 2012. Éléments pour une « archéologie » de la méthode directe. Documents pour l'histoire du français langue étrangère ou seconde. URL : http://journals.openedition.org/dhfles/3386

Birdsong, David. 2007. Nativelike pronunciation among late learners of French as a second language. In Ocke-Swen Bohn \& Murray J. Munro (éds.), Language 
Experience in Second Language Learning. In Honor of James Emil Flege. Amsterdam/Philadelphie : John Benjamins, 99-16.

Bødtker, A.T. \& Høst, Sigurd. 1896. Løerebog i fransk for begyndere. Kristiania : Grimsgaard \& Malling.

Cadre européen commun pour les langues. 2018. URL : https://rm.coe.int/cecrvolume-complementaire-avec-de-nouveaux-descripteurs/16807875d5

Clopper, Cynthia G. \& Pisoni, David B. 2004. Effects of talker variability on perceptual learning of dialects. Language and Speech 47:3, 207-239.

Delatour, Y., Jennepin, D., Léon-Dufour, M., Mattlé, A. \& Teyssier, B. 1991. Grammaire du français. Paris : Hachette FLE.

Detey, Sylvain. 2009. Phonetic input, phonological categories and orthographic representations: a psycholinguistic perspective on why oral language education needs oral corpora. The case of French-Japanese interphonology development. In Yuji Kawaguchi, Makoto Minegishi \& Jacques Durand (éds.), Corpus Analysis and Variation in Linguistics. Amsterdam/Philadelphie : John Benjamins, 179-200.

Detey, Sylvain, Durand, Jacques, Laks, Bernard \& Lyche, Chantal (éds.). 2010. Les variétés du français parlé dans l'espace francophone. Ressources pour l'enseignement. Paris : Ophrys.

Detey, Sylvain, Durand, Jacques, Laks, Bernard \& Lyche, Chantal (éds.). 2016. Varieties of Spoken French. Oxford: Oxford University Press.

Detey, Sylvain \& Lyche, Chantal. 2019. Enseigner la variation diatopique en FLE dans deux contextes L1 variationnels distincts : le cas de la Norvège et du Japon. In Laurent Gajo, Jean-Marc Luscher, Isabelle Racine \& Françoise Zay (éds.), Variation, plurilinguisme et évaluation en français langue étrangère. Berlin : Peter Lang, 47-59.

Detey, Sylvain \& Racine, Isabelle. 2012. Les apprenants de français face aux normes de prononciaiton : quelle(s) entrée(s) pour quelle(s) sortie(s) ? Revue française de linguistique appliquée 17 : 1, 81-96.

Durand, Jacques, Laks, Bernard \& Lyche, Chantal. 2009. Le projet PFC (phonologie $d u$ français contemporain : une source de données primaires structurées. In Jacques Durand, Bernard Laks \& Chantal Lyche (éds), Phonologie, variation et accents du français. Paris : Hermès, 19-62. 
Durand, Jacques \& Lyche, Chantal. 2003. Le projet 'Phonologie du français contemporain' et sa méthodologie. In Elisabeth Delais-Roussarie \& Jacques Durand (éds.), Corpus et variation en phonologie du français. Toulous : Presses Universitaires du Mirail, 213-276.

Durand, Jacques \& Lyche, Chantal. 2019. Paul Passy, Johan Storm and the palatal nasal in modern French. In Jan Kristian Hognestad, Torodd Kinn \& Terje Lohndal (éds.), Fonologi, sosiolingvistikk og vitenskapsteori. Festskrift til Gjert Kristoffersen. Oslo : Novus, 79-97.

Durand, Jacques \& Lyche, Chantal. Sous presse. Retour sur Les sons du français : la modernité de Passy. Journal of French Languages Studies.

Falkert, Anika. 2019. La place de la variation dans l'enseignement de la phonétique en FLE. Recherches en didactique des langues et des cultures. URL : http://journals.openedition.org/rdlc/4309; DOI : 10.4000/rdlc.4309.

Fouché, Pierre. 1959. Traité de prononciation française. Paris : Klincksieck.

Gadet, Françoise. 2007. La variation sociale en français. Paris : Ophrys.

Galazzi, Enrica. 2015. La prononciation du français standard à l'épreuve du troisième millénaire. Repères DoRiF N.8 - Parcours variationnels du français contemporain, DoRiF Università, Roma septembre 2015, http://www.dorif.it/ezine/ezine_articles.php?id=235

Germain, Claude. 1993. Évolution de l'enseignement des langues : 5000 ans d'histoire. Paris : CLE international.

Gooskens, Charlotte \& Heeringa, Wilbert. 2014. The role of dialectal exposure in receptive multilingualism. Applied Linguistics Review 5:1, 247-271.

Grammont, Maurice. 1914. La prononciation française. Traité pratique. Paris : Delagrave.

Halté, Jean-François. 1992. La didactique du français. Paris : PUF.

Henriksen, Turid \& Lyche, Chantal. 1997. L'enseignement de la prononciation du français en Norvège dans la première moitié du 20e siècle : évolution des stratégies. Documents du français langue étrangère ou seconde 19, 206-220.

Henriksen, Turid. 2004. Begynnerbøker i fransk fra 1896 til 1974. A. Trampe Bødtker og Sigurd Høst, Lorebog i fransk for begyndere og dens arvtakere. Innhold, tekststrategier og iscenesettelser. Thèse de doctorat. Université d'Oslo 
Howatt, Anthony P.R. \& Smith, Richard C. 2002. Modern Language Teaching. The Reform Movement. 5 volumes. London/New York : Routledge.

Howatt, Anthony P.R \& Widdowson, H.G. 2004. A History of English Language Teaching. $2^{\text {ème }}$ éd. Oxford : OUP.

Husby, Olav. 2015. Andrespråk og uttale. In Kristin Melum Eide (éd.), Norsk andrespråkssyntaks. Oslo: Novus Forlag, 325-400.

Jespersen, Otto. 1904. How to Teach a Foreign Language. London: Swann Sonnenschen \& Co. Ltd.

Koschwitz, Eduard. 1896. Les parlers parisiens. Anthologie phonétique. $2^{\text {ème }}$ éd. H. Welter.

Labov, William. 1966. The Social Stratification of English in New York City. Washington : Center for Applied Linguistics.

Labov, William. 1972. Sociolinguistic Patterns. Philadelphia : University of Pennsylvania Press.

Laks, Bernard. 2002. Description de l'oral et variation : la phonologie et la norme. L'information grammaticale 94, 5-11.

Ledegen, Gudrun. 2000. Le bon français. Paris : L'Harmattan.

Lively Scott E., Logan, John S. \& Pisoni, David B. 1993. Training Japanese listeners to identify English $/ \mathrm{r} /$ and $/ \mathrm{l} / \mathrm{:}$ the role of phonetic environment and talker variability in learning new perceptual categories. Journal of the Acoustical Society of America 94, 1242-1255.

Martinet, André \& Walter, Henriette. 1973. Dictionnaire de la prononciation française dans son usage réel. Paris : France-Expansion.

Morin, Yves-Charles. 2000. Le français de référence et les normes de pronunciation. Cahiers de l'Institut de linguistique de Louvain $26: 1,91-135$.

Nyrop, Kristoffer. 1893. Kortfattet fransk lydloere. Til bruk for loerere og studerende. København : P.G. Philipsens Forlag.

Nyrop, Kristoffer. 1902. Manuel phonétique du français parlé. Deuxième édition traduite et remaniée par Emanuel Philipot. København : Det nordiske Forlag.

Passy, Paul. 1887, 1889, 1892, 1895, 1899, 1906, 1913, 1917, 1922, 1925, 1929, 1932. Les sons du français. Paris : Librairie Firmin-Didot. 
Passy, Paul. 1914. A French Phonetic Reader. London : University of London Press.

Paternostro, Roberto. 2017. Peut-on enseigner la variation? In Henry Tyne, Mireille Bilger, Paul Cappeau \& Emmanuelle Guérin (éds.), La variation en question. Hommages à Françoise Gadet. Bruxelles : Peter Lang, 279-290.

Puren, Christian. 1988. Histoire des méthodologies de l'enseignement des langues. Paris : Nathan CLE international.

Slabakova, Roumyana. 2013. Adult second language acquisition. A selective overview with focus on the learner linguistic system. Linguistic Approaches to Bilingualism $3: 1,48-72$.

Storm, Johan. 1879. Engelsk Filologi. Anvisning tilt il et videsnkabeligt Studium af det engelske Sprog for Studerende, Lerere og Viderekomme. I: Det levende Sprog. Kristiania : Alb. Cammermeyer (Det mallingske Bogtrykkeri).

Sweet, Henry. 1899. The Practical Study of Languages. A guide for teachers and learners. London : J:M: Dent \& Co.

Thomson, Ron I. 2012. Improving L2 listeners' perception of English vowels: A computer mediated approach. Language Learning $62: 4,1231-58$.

Valdman, Albert. 2000. Comment gérer la variation dans l'enseignement du français langue étrangère aux Etats-Unis. The French Review 73 : 4, 648-666.

Wachs, Sandra. 2017. Écriture numérique spontanée et variabilité. Un écrit-oral à exploiter en français langue étrangère (sensibiliser aux styles et à la prononciation). In Henry Tyne, Mireille Bilger, Paul Cappeau \& Emmanuelle Guérin (éds.), La variation en question. Hommages à Françoise Gadet. Bruxelles : Peter Lang, 237-254.

Weber, Corinne. 2019. Interrogations épistémologiques autour de l'oralité, Recherches en didactique des langues et des cultures 16-1. URL : http://journals.openedition.org/rdlc/4252; DOI : 10.4000/rdlc.4252

Østby, Kathrine Asla. 2016. Les voyelles orales à double timbre dans le parler de la haute bourgeoisie parisienne : analyse acoustique et diachronique. Thèse de doctorat. Université d'Oslo et Université de Paris Ouest Nanterre La Défense. 
COORDONNÉES

Chantal Lyche

Université d'Oslo

chantal.lyche@ilos.uio.no

Kathrine Asla Østby

Université d'Oslo

k.a.ostby@ilos.uio.no 